FORMATION Formation emploi

Revue française de sciences sociales

98 | avril-juin 2007

Pour une approche par les capacités

\title{
Introduction : À la recherche de principes novateurs pour l'action publique
}

Robert Salais

\section{OpenEdition}

1 Journals

Édition électronique

URL : http://journals.openedition.org/formationemploi/1548

DOI : 10.4000/formationemploi.1548

ISSN : 2107-0946

Éditeur

La Documentation française

Édition imprimée

Date de publication : 1 avril 2007

Pagination : 5-8

ISSN : 0759-6340

\section{Référence électronique}

Robert Salais, «Introduction : À la recherche de principes novateurs pour l'action publique », Formation emploi [En ligne], 98 | avril-juin 2007, mis en ligne le 19 février 2009, consulté le 30 octobre 2020. URL : http://journals.openedition.org/formationemploi/1548; DOI : https://doi.org/10.4000/

formationemploi. 1548

(c) Tous droits réservés 


\title{
Numéro spécial
}

\section{Introduction : \\ À la recherche de principes novateurs pour l'action publique}

\author{
Par Robert Salais*
}

Ce numéro spécial de Formation Emploi, consacré à l'approche par les capacités, témoigne du progrès des idées et recherches d'Amartya Sen dans la communauté des chercheurs français et internationaux. Ces idées et recherches débordent les domaines de la pauvreté et du sous-développement, où elles sont de longue date appliquées, pour entrer dans ceux de l'emploi, de la politique sociale et du développement économique (à distinguer de la croissance) des pays riches. C'est un premier point à souligner. Le second point qui doit être signalé est que ce numéro témoigne d'une insatisfaction croissante de cette même communauté devant les principes qui guident actuellement l'action publique en matière d'emploi, de formation et de marché du travail, ainsi que devant leurs résultats. Il existe en effet un divorce de plus en plus visible entre l'accroissement apparent des performances quantitatives des politiques d'emploi et le développement des emplois précaires et mal payés, celui des parcours sans issue ou de tensions difficilement supportables entre la vie désirée et le travail accessible, pour une part appréciable de la population. Ces problèmes incitent à la recherche d'autres principes d'observation et d'action (en un mot, d'évaluation), d'où l'intérêt pour l'approche par les capacités. Car celle-ci propose et applique un autre principe d'évaluation des situations de vie et de travail que la performance globale. Pour le dire simplement, elle se concentre sur la mesure des possibilités réellement accessibles pour chaque personne en matière de vie et de travail. Traduite en objectifs des politiques d'emploi ou de formation, une telle «métrique» implique de lutter contre les inégalités en termes d'accès à des possibilités considérées comme de valeur: par exemple, qualité de l'emploi, sécurité professionnelle, réelle conciliation entre vie privée et vie professionnelle, possibilités de promotion et de mobilité, possibilités de s'engager dans des projets de long terme (fonder une famille, avoir des enfants...).

L'approche par les capacités ouvre ainsi un chantier de recherche ambitieux, innovateur et motivant dans de multiples domaines: méthodes et instruments empiriques, fondements théoriques, modélisations,

* Robert Salais, économiste, est membre de l'UMR IDHE (Institutions et Dynamiques Historiques de l'Économie). À la suite du programme de recherche européen EUROCAP, consacré à l'approche par les capacités, il coordonne le projet intégré CAPRIGHT du bè Programme-Cadre européen. Ses recherches portent sur l'analyse économique des conventions et l'approche par les capacités appliquée aux politiques d'emploi. Voir R. Salais et R. Villeneuve, Éds., 2004, Europe and the politics of capabilities, Cambridge, Cambridge University Press. 
évaluation des politiques publiques, ainsi qu'à différents niveaux : parcours individuels ; organisation des entreprises ; formation initiale, générale, professionnelle et continue; inscription territoriale des politiques d'emploi, de formation ou sociales ; définition et évaluation des politiques nationales. En commençant à soutenir des programmes de recherche consacrés à cette approche, l'Union européenne prend conscience, à ce jour encore à la marge, du besoin de renouveler et d'étendre sa palette de réflexions sur le social, parent pauvre de la construction européenne.

Bien évidemment les difficultés à résoudre, les questions à approfondir ne manquent pas lorsqu'il s'agit de mettre en œuvre une idée nouvelle. C'est à elles que s'attaquent les contributions rassemblées dans ce numéro. Un des éléments les plus remarquables est la diversité des horizons et des voies par lesquelles leurs auteurs sont arrivés jusqu'aux travaux d'Amartya Sen, et à l'approche par les capacités, et ont découvert les potentialités qu'ils offraient pour bâtir leur questionnement et interpréter leurs résultats. Chaque auteur y va de sa lecture plus ou moins approfondie, de ce qu'il a compris et de ce qu'il a retenu pour conduire à bien son travail sur son objet. Tous combinent une présentation des travaux de Sen qui les inspirent avec le traitement de matériaux empiriques spécifiques. Cette diversité n'occulte pas, bien au contraire, un ensemble de préoccupations communes sur lesquelles nous voudrions insister au risque d'oublier, de simplifier ou de trahir partiellement la pensée de certains auteurs. On aboutit ainsi à une masse critique de travaux, de méthodologies, de questions de recherche, de résultats et d'interrogations. Ce dossier devrait constituer un passage obligé pour les recherches futures.

Les trois premières contributions offrent une présentation approfondie de l'approche par les capacités, respectant l'esprit (et la lettre) des travaux d'Amartya Sen. Ceci vient, à notre sens, de ce que les auteurs sont engagés dans des programmes collectifs de recherche, l'un en Europe, l'autre en Nouvelle-Zélande ${ }^{1}$, qui se sont fixé pour objet de se réapproprier la démarche et de la développer dans le domaine de l'emploi, de l'entreprise et du travail.

${ }^{1}$ Il s'agit, pour l'Europe, des programmes EUROCAP et CAPRIGHT et pour la Nouvelle-Zélande du programme « Développement des capacités humaines: institutions de l'emploi, organisations et individus ».
Jean-Michel Bonvin et Nicolas Farvaque relèvent l'ambivalence (notion que l'on retrouve dans plusieurs autres contributions) des politiques de lutte contre le chômage (et de leur volet formation) promues par l'OCDE (Organisation de coopération et de développement économiques) et l'Union européenne. Cette ambivalence est manifeste dans la référence à l'employabilité. S'agit-il de formater les individus et de les sélectionner en fonction des standards de productivité en vigueur ou de les aider à s'intégrer durablement et à trouver leur place dans la société ? Ils montrent comment l'approche par les capacités permet de lever cette ambivalence, de clarifier la diversité des fondements normatifs et des mises en œuvre sur le terrain. Les enjeux portent sur l'interprétation de la liberté réelle de choix chez Sen, sur le rôle et le choix de la base informationnelle de jugement (c'est-à-dire le choix des variables et des critères jugés pertinents pour l'action publique), sur les interactions processuelles entre liberté réelle, participation démocratique et responsabilité individuelle. Disposer d'une liberté réelle n'est pas, pour Sen, avoir un contrôle individuel de son choix (conception individualiste), mais disposer d'une capacité effective à être et à faire grâce à laquelle la personne peut choisir librement entre différentes opportunités de valeur.

Comme le soulignent Delphine Corteel et Bénédicte Zimmermann dans leur contribution, la capacité est, avant tout, un pouvoir d'agir. La liberté, rappellentelles, est une valeur du développement humain, associée à l'accomplissement personnel. Cette perspective implique des politiques publiques tendues vers la « capacitation» des personnes, et une participation de ces dernières aux processus d'élaboration, de mise en œuvre et d'évaluation des politiques. L'analyse de l'organisation et des politiques des entreprises se déplace de la notion de compétences (notion essentiellement instrumentale liée à des besoins de flexibilité et d'adaptabilité) vers celle de capacités. L'enquête sur les capacités implique, pour les auteurs, de se centrer sur les parcours biographiques et les contextes organisationnels. Les tournants et les bifurcations de parcours ainsi que les critères d'évaluation et d'accès aux ressources dans ces moments clés sont de puissants révélateurs des structures d'opportunités. La méthode permet d'observer, par exemple en cas de restructuration, l'absence de 
contenu des formations adéquat, à la fois au problème et aux personnes. Il devient essentiel en ces moments, pour les salariés et leurs représentants, d'avoir prise sur l'évaluation et l'accès aux ressources, en particulier pour écarter la sélection des bénéficiaires sur des critères abstraits, tels que l'âge ou le diplôme, sur lesquels ils ne peuvent rien.

La contribution de Jane Bryson et Kiri Merritt recoupe certaines conclusions de Delphine Corteel et Bénédicte Zimmermann d'une manière étonnante, compte tenu des différences de contexte- la Nouvelle-Zélande versus la France - et de question en quoi l'activité de travail agit-elle comme moteur, contribution ou frein au développement des capacités ? Elles repèrent dans leurs entretiens semistructurés le rôle déclenchant de certains évènements ou caractéristiques de la situation (l'encouragement donné par certaines personnes de l'encadrement ou de l'environnement proche ; la création d'une atmosphère locale d'initiative et de liberté). Elles aussi séparent les notions de management (capital humain, performance) de celle de capacité humaine (human capability). Seule l'approche par les capacités, au lieu d'en faire une sphère isolée, intègre le travail dans la vie et reconnait qu'il absorbe une part importante de son temps. Du coup apparaît l'extraordinaire interconnexion des différents et multiples moments de la vie : ce qui se passe dans l'un a des effets dans l'autre (un moment de travail sur un moment de vie, et réciproquement). L'objectif de qualité de l'emploi, ajouterons-nous, doit partir de cette imbrication et exiger, pour être correctement construit, la participation des intéressés.

La réforme de l'apprentissage en « unités standards » en Nouvelle-Zélande fait écho aux logiques de performance que signalent les contributions de JeanMichel Bonvin et Nicolas Farvaque, de Delphine Corteel et Bénédicte Zimmermann mais aussi de Marie Verhoeven, Jean-François Orianne et Vincent Dupriez. Ces derniers s'interrogent sur le mouvement de réforme en Belgique des politiques d'éducation. Va-t-on vers des politiques « capacitantes »? La matière historique est vaste et se prête plus ou moins aisément au décryptage fin qu'implique, si l'on suit les auteurs précédents, l'approche par les capacités. La mise en contexte historique, de même que l'exemple contemporain de la Guinée Maritime (contribution de Jean-Étienne Bidou et Isabelle Droy), fait apparaître que l'accès au savoir formel dépend, pour l'enfant, du fait de pouvoir aller à l'école et de pouvoir y rester. La gratuité et l'obligation scolaire ne peuvent donc être qualifiées de droits purement formels. Ce sont les premières mesures nécessaires à une effectivité du droit à l'éducation. Sans elles, pas d'accès de tous au savoir. Il n'y a donc rien de strictement utilitariste, ni de ressourciste là-dedans. L'idéal d'égalité de résultats et l'approche de l'éducation par les compétences doivent plus, comme le suggèrent les auteurs, à la diffusion du New Public Management ${ }^{2}$ par l'OCDE qu'à la prise de conscience par les décideurs des vertus de l'approche par les capacités.

Bien entendu, toute politique est traversée de logiques contradictoires et les réflexions en cours en Belgique sur l'égalisation des contextes d'éducation se prêteraient davantage à une prise en compte de l'inégalité des capacités. Car, contrairement aux approches structuralistes qui réduisent les régularités statistiques à des contraintes indépassables, une situation éducative n'est jamais complètement telle qu'elle parait être. La contribution de Marie de Besses sur l'action éducative en lycée professionnel souligne avec justesse que l'émergence de possibilités et leur accès dépendent de facteurs tels que l'estime de soi, la capacité à se projeter dans l'avenir, la prise de conscience que les contraintes sont aussi parfois des ressources si on apprend à formuler des objectifs. Comme le révèlent les entretiens, l'émergence de ces facteurs est très liée à la qualité du contexte éducatif, en particulier à la vie collective de l'établissement, aux relations entre les acteurs, à la conception de l'orientation professionnelle. Ces résultats confirment qu'un même dispositif formel, appuyé sur des ressources identiques, peut conduire à une conversion très inégale en résultats de valeur. Participation, délibération, attention à la singularité des parcours et, au fond, confiance des participants

\footnotetext{
${ }^{2}$ L'école (diversifiée en son sein) du New Public Management importe, dans le domaine des politiques publiques, des technologies de pilotage par la performance (laquelle est en général mesurée par des indicateurs) issues du management de l'entreprise. Toute réforme est bonne quels que soient les moyens utilisés, du moment qu'elle accroît la performance à coût constant. Contrairement à l'approche par les capacités, ces technologies laissent de côté les fondements de l'action publique : réalisation du bien commun, référence à des principes de justice, choix démocratique des objectifs et des modes d'évaluation.
} 
dans les possibilités de la situation sont des paramètres importants dans la recherche d'une égalisation des contextes. Ils influent considérablement sur la construction de la base informationnelle de jugement sur l'élève, en l'ouvrant ou non à la prise en compte des «traits focaux $»^{3}$ (concept d'Amartya Sen) pertinents de sa situation.

La contribution de Jean-Etienne Bidou et Isabelle Droy provient du domaine « classique », où les perspectives ouvertes par Amartya Sen sont bien connues et travaillées. Il n'en est que plus intéressant de retrouver des problèmes communs avec les contributions précédentes, même s'ils sont formulés dans des contextes différents. Car la problématique s'évade des approches habituelles (et fatalistes) en termes de pauvreté pour aborder l'activité, les savoirs, l'apprentissage. La contribution souligne spécialement les inégalités de sexe, la valeur intrinsèque de certains fonctionnements de base (comme être en bonne santé, suivre durablement l'école) dans l'accès au savoir formel. Les trois groupes de facteurs de conversion (personnels, d'environnement, institutionnels) sont bien mis en évidence, ce qui permet d'échapper à la vision individualiste des inégalités de capacités. Un peu comme chez Delphine Corteel et Bénédicte Zimmermann, l'enquête s'appuie sur un dispositif à plusieurs étages, en l'occurrence : sélection de sites d'enquête, enquête exhaustive sur les ménages de ces sites (dite «base pauvreté »), enquête sur un sous-échantillon d'individus (base «multi-activité»), enfin collecte de récits de vie pour une partie raisonnée d'entre eux.

Les contributions de Marion Lambert et Josiane Vero, et de Tristan Klein et Christine Le Clainche mettent certains dispositifs des politiques d'emploi et de formation à l'épreuve de l'approche par les capacités, l'accès à la formation continue d'une part, le contrat initiative emploi (CIE) et le contrat emploi solidarité (CES) d'autre part. Plus encore que les précédentes, elles soulèvent la question de la manière de rendre opérationnelle l'approche par les capacités. Toutes deux recourent à des données individuelles (qui n'ont été de plus que partiellement ou pas du tout prévues pour cette approche). Il en ressort une difficulté à dépasser une conception individualiste des capacités (ce qui n'est en aucun cas, bien sûr, une critique de l'excellente qualité technique de ces travaux). D'où trois questions auxquelles s'affrontent les contributions : comment séparer, dans l'évaluation que fait une personne de sa situation, ce qui relève d'un jugement objectif ou d'une adaptation subjective (en général vers le bas) à des contraintes qu'elle juge inéluctables (préférences adaptatives)? Comment éviter de naturaliser des catégories générales (telles que le sexe, l'âge ou le niveau de formation ou de qualification) et d'en faire des déterminants structurels, voire des propriétés intrinsèques des individus? Comment évaluer l'efficacité d'une mesure si on est obligé, faute de données sur la situation venant d'autres sources, d'employer les catégories et variables employées par cette mesure pour définir les conditions d'accès, les ressources, les droits ? Car ce sont ces catégories et variables institutionnelles dont il faudrait pouvoir apprécier l'effet de manière indépendante. Une réponse commune à ces questions est que le chercheur doit pouvoir disposer de données sur un même échantillon de situations provenant de différentes méthodologies : données de panel; enquêtes multi-étages à la fois objectives et subjectives, quantitatives et qualitatives. Le spectre de ces sources et méthodes est parcouru dans ce dossier. Il reste dans le futur, parmi d'autres tâches, à en examiner la combinatoire.

\footnotetext{
${ }^{3}$ Les traits focaux (ou focal features) sont les caractéristiques empiriques qui s'avèrent pertinentes pour rendre compte de l'inégalité des capacités entre les personnes. Ainsi, dans l'exemple, ce sont les variables les mieux à même de restituer la qualité du contexte éducatif, appréciée du point de vue de l'élève (accès à l'estime de soi ou capacité à se projeter dans l'avenir).
} 